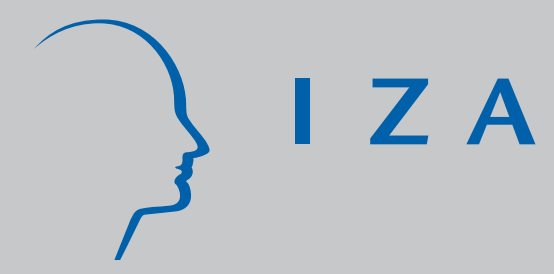

IZADP No. 2128

Learning from a Piece of Pie:

The Empirical Content of Nash Bargaining

Pierre-André Chiappori

Olivier Donni

April 2006 


\title{
Learning from a Piece of Pie: The Empirical Content of Nash Bargaining
}

\author{
Pierre-André Chiappori \\ Columbia University \\ Olivier Donni \\ THEMA, University of Cergy-Pontoise \\ and IZA Bonn
}

Discussion Paper No. 2128

April 2006

IZA

P.O. Box 7240

53072 Bonn

Germany

Phone: +49-228-3894-0

Fax: +49-228-3894-180

Email: iza@iza.org

Any opinions expressed here are those of the author(s) and not those of the institute. Research disseminated by IZA may include views on policy, but the institute itself takes no institutional policy positions.

The Institute for the Study of Labor (IZA) in Bonn is a local and virtual international research center and a place of communication between science, politics and business. IZA is an independent nonprofit company supported by Deutsche Post World Net. The center is associated with the University of Bonn and offers a stimulating research environment through its research networks, research support, and visitors and doctoral programs. IZA engages in (i) original and internationally competitive research in all fields of labor economics, (ii) development of policy concepts, and (iii) dissemination of research results and concepts to the interested public.

IZA Discussion Papers often represent preliminary work and are circulated to encourage discussion. Citation of such a paper should account for its provisional character. A revised version may be available directly from the author. 


\section{ABSTRACT \\ Learning from a Piece of Pie: The Empirical Content of Nash Bargaining*}

Consider a model of bargaining, in which two players, 1 and 2, share a pie of size $y$. The bargaining environment is described by a set of parameters $\lambda$ that may affect agents' preferences over the agreement sharing, the status quo outcome, or both. The outcomes (i.e., whether an agreement is reached, and if so the individual shares) and the environment (including the size of the pie) are known, but neither the agents' utilities nor their threat points. Assuming that the agents adopt a Nash bargaining solution, we investigate the empirical content of this assumption. We first show that in the most general framework, any outcome can be rationalized as a Nash solution. However, if (i) the size of the pie $y$ does not influence the players' threat points and (ii) there exist (at least) two parameters $\lambda_{1}$ and $\lambda_{2}$ that are player-specific, in the sense that $\lambda_{\mathrm{i}}$ does not influence the utility or the threat point of player $j \neq i$, then Nash bargaining generates strong testable restrictions. Moreover, the underlying structure of the bargaining, i.e., the players' utility and threat point functions, can be recovered under slightly more demanding conditions.

\section{JEL Classification: $\quad$ C71, C78}

Keywords: bargaining game, Nash solution, testability, identifiability, cardinal utility

Corresponding author:

Olivier Donni

University of Cergy-Pontoise

33, Boulevard du Port

95011 Cergy-Pontoise Cedex

France

Email: olivier.donni@eco.u-cergy.fr

\footnotetext{
* Paper presented at seminars in Chicago and Paris. We thank participants, and in particular Jaap Abbring, Bernard Fortin and Jim Heckman for useful comments and suggestions. Financial support from the NSF (grant) and the FQRSC is gratefully acknowledged. Errors are ours.
} 
I passed by his garden, and marked, with one eye, How the Owl and the Panter were sharing a pie:

The Panther took pie-crust, and gravy, and meat, While the Old had the dish as its share of the treat. When the pie was all finished, the Owl, as a boon, Was kindly permitted to pocket the spoon:

While the Panther received knife and fork with a growl, And concluded the banquet by...

Lewis Caroll (Alice's Adventures in Wonderland, 1866)

\section{Introduction}

Consider an experiment in which two agents, 1 and 2, bargain about a pie of size $y$. If the agents agree on some sharing $\left(\rho_{1}, \rho_{2}\right)$ with $\rho_{1}+\rho_{2}=y$, it is implemented. If not, each agent $i$ receives some reservation payment $\lambda_{i}$. Information is complete: each agent knows his opponent's preferences as well as the structure of the game. The interaction is repeated for different pie sizes and different reservation payments. The outcomes (i.e., whether an agreement is reached, and if so the individual shares), as functions of the size of the pie and the payments $\lambda_{1}, \lambda_{2}$, are observable by an outside econometrician; however, individual utilities are not. Assume, finally, that the econometrician has a prior theory about the agents' behavior; specifically, she believes that it can be described using the concept of Nash bargaining (Nash, 1950). Is this theory testable (i.e., is there a particular set of possible outcomes that would violate the Nash bargaining property), or is it the case that any outcome can be rationalized by a Nash-bargaining setting for wellchosen individual preferences? And how much can be learned about the true structure of the model (i.e., the utility individuals derive from the consumption of either their share of the pie or their reservation payment) from the sole observation of the outcomes? These two questions - the testability of Nash bargaining models and the identifiability of their underlying structure from observed behavior — are the main topic of this paper.

A possible approach, which is often adopted in the empirical literature on Nash bargaining, is to answer the first question by ignoring the second. Indeed, many works arbitrarily assume a specific (usually linear) form for individual utilities. Then the sharing function $\left(\rho_{1}=\rho, \rho_{2}=y-\rho\right)$ solves the 
program:

$$
\max _{\rho}\left(\rho-\lambda_{1}\right)\left(y-\rho-\lambda_{2}\right),
$$

giving the simple, linear form $\rho=\frac{1}{2} \lambda_{1}+\frac{1}{2} y-\frac{1}{2} \lambda_{2}$. Convenient as it may be, this solution totally relies on the linearity assumption; since the Nash bargaining outcome depends on the cardinal representation of individual preferences, any deviation from linear utilities will give a different form for the resulting shares. ${ }^{1}$ This feature is clearly problematic, since the linearity assumption is generally made for convenience rather than realism or theoretical consistency. ${ }^{2}$ In particular, any test based on the form (1) is a joint test of two assumptions, one general (Nash bargaining), the other very specific (linear utilities). A rejection is likely to be considered as inconclusive, since the burden of rejection can always be put on the specific and often ad hoc linearity assumption. For that reason, we believe preferable to adopt the generally accepted rule in empirical economics, whereby preferences should be recovered from the data rather than assumed a priori.

In the present paper, we address these issues in a general framework where the environment is described by a set of parameters that may affect agents' preferences over the agreement sharing, the status quo outcome, or both. A key role will be played by the econometrician's prior information on the structure of the model at stake. In a non-parametric spirit, this information will be described by some (broad) classes to which the utility or threat point functions are known to belong. We are mainly interested in situations in which this prior information is limited. We thus do not assume that the econometrician knows the parametric form of the utility and threat point functions, but simply that these functions are known to satisfy some exclusion restrictions. ${ }^{3}$ Our basic question can thus be precisely restated in the following way: what is the minimum prior information needed to achieve (i) testability of the Nash bargaining theory, and (ii) identifiability of the underlying structural model.

Regarding the identifiability issue, an interesting aspect is that Nash so-

\footnotetext{
${ }^{1}$ In the exemple above, for instance, if the utility of agent 1 is $U(x)=\sqrt{x}$ instead of $U(x)=x$, the solution becomes $\rho=\frac{1}{3} y+\frac{2}{9} \lambda_{1}-\frac{1}{3} \lambda_{2}+\frac{2}{9} \sqrt{\lambda_{1}\left(\lambda_{1}+3 y-3 \lambda_{2}\right)}$.

${ }^{2}$ See Farber (1986, pp. 1056-57) for a discussion of the linear utility framework in the context of union-firm bargaining.

${ }^{3}$ To put it in a Popperian perspective: we do not want the falsifiability of Nash bargaining to be entirely driven by ad hoc auxiliary hypotheses — such as particular functional forms of individual utility functions.
} 
lutions are not invariant to monotonic transformations of utility functions. It follows that one may, in principle, try to retrieve a cardinal representation of preferences. While the identification of cardinal preferences is a standard problem in economics, our approach is original in that it does not involve uncertainty. Whether concavity of utility functions matter in bargaining because of risk aversion (as might be suggested by the non cooperative foundations of Nash bargaining) or for unrelated reasons is an interesting conceptual problem, on which our findings shed a new light.

The empirical content of game theory is undoubtedly a topical issue as illustrated by several recent contributions. For example, Sprumont (2001) considers, from the revealed preferences viewpoint, a non-cooperative game played by a finite number of players, each of whom can choose a strategy from a finite set. Ray and Zhou (2001) adopt a similar set-up but focuses on extensive-form games. Other related papers include Bossert and Sprumont (2002, 2003), Carvajal (2002), Carvajal, Ray and Snyder (2004), Zhou (1999, 2002), Ray and Snyder (2003), Xu and Zhou (2004). Nonetheless, our contribution differs in many respects from what is generally made. Firstly, our subject matter — the Nash solution - has never been investigated in spite of the various applications of bargaining models in economics. ${ }^{4}$ Secondly, our methodology is not based on revealed preferences. The inspiration of the present paper, in fact, is more closely related to the work of Chiappori (1988, 1992) and its numerous sequels (Chiappori and Ekeland, 2003, 2004), on the empirical implications of Pareto efficiency. This methodology is probably more appropriate for the empirical implementation of theoretical results. Thirdly, the emphasis of this paper is largely on the identification problem, which is generally ignored by the authors cited above.

The organization of the paper is as follows. In the next Section, we develop the general model and show that neither testability, nor identification obtain without a priori information on utility and threat point functions. In Section 3, then, we introduce additional structure into the model, and show that testability obtains under mild assumptions on utility and threat point functions. In Section 4, we note that identification requires stronger assumptions, of which several examples are given. We show in particular that in the simple example given above, identification obtains provided that the

\footnotetext{
${ }^{4}$ See, for example, the applications of bargaining theory in models of household behavior, trade-union negotiations, experimental economics, job matching/search, international trade, oligopolistic competition.
} 
utility functions do not belong to the exponential family. In Section 5, the possibility of disagreement between players is considered and some additional restrictions on observable behavior are derived. In the concluding Section, we discuss the potential applications of the results.

\section{The general model}

\subsection{The framework}

We consider a bargaining game where two players, 1 and 2, share a pie of size $y$. The bargaining environment is described by a vector $\lambda$ of $l$ parameters. The outcome of the bargaining game, as a function of the size of the pie and the parameters, is observed. Specifically, we assume that the relevant parameters $(y, \lambda)$ vary within some convex, compact subset $\mathcal{S}$ of $\mathbb{R}_{+} \times \mathbb{R}^{l}$. Let $\mathcal{N}$ denote the subset of $\mathcal{S}$ on which one observes that no agreement is reached (so that agents receive their reservation payment), and $\mathcal{M}=\mathcal{S}-\mathcal{N}$ its complement. ${ }^{5}$ Over $\mathcal{M}$, a sharing is observed, in which player $i$ gets $\rho_{i}(y, \lambda)$, with $\rho_{1}(y, \lambda)+\rho_{2}(y, \lambda)=y$. For notational convenience, we define the sharing function $\rho$ as the share of the pie allocated to member 1, i.e., $\rho(y, \lambda)=\rho_{1}(y, \lambda)$ (then $y-\rho(y, \lambda)=\rho_{2}(y, \lambda)$ ); it is natural to assume that $\rho(y, \lambda) \in[0, y]$ for all $(y, \lambda) \in \mathcal{S}$. The agents' observed behavior is defined by the partition $\{\mathcal{M}, \mathcal{N}\}$ of $\mathcal{S}$ and the function $\rho(y, \lambda)$ defined over $\mathcal{M}$. Let $U^{i}\left(\rho_{i}, \lambda\right)$ denote $i$ 's utility when an agreement is reached and the sharing $\rho$ is implemented. ${ }^{6}$ Similarly, let $T^{i}(y, \lambda)$ denote $i$ 's threat point, i.e., utility when no agreement is reached and the reservation payments are made. The functions $U^{i}\left(\rho_{i}, \lambda\right)$ and $T^{i}(y, \lambda)$ may be, in general, different. We assume throughout the paper that these functions have the following regularity properties.

\section{Assumption S1}

(a) The functions $U^{i}\left(\rho_{i}, \lambda\right)$ are strictly increasing and concave in $\rho_{i}$.

\footnotetext{
${ }^{5}$ If the players are indifferent between the agreement and the disagreement outcomes, we adopt the convention that they always choose the agreement outcome.

${ }^{6}$ Note, in particular, that each player's utility (in case of an agreement) depends on his share and on the set of environmental parameters, but neither on the other player's share nor on the initial size of the pie.
} 
(b) The functions $U^{i}\left(\rho_{i}, \lambda\right)$ and $T^{i}(y, \lambda)$ are three times continuously differentiable in all their arguments..

The set of all functions $U^{i}\left(\rho_{i}, \lambda\right)$ (resp. $\left.T^{i}(y, \lambda)\right)$ that are compatible with the a priori knowledge of the econometrician is denoted by $\mathfrak{U}^{i}$ (resp. $\mathfrak{T}^{i}$ ) and $\Omega=\mathfrak{U}^{1} \times \mathfrak{U}^{2} \times \mathfrak{T}^{1} \times \mathfrak{T}^{2}$ is the information set of the econometrician. We first introduce the following definition.

Definition 1 Suppose a given set of prior information $\Omega$. The agents' (observed) behavior $(\{\mathcal{M}, \mathcal{N}\}, \rho)$ is compatible with Nash bargaining if and only if there exist two utility functions $U^{i} \in \mathfrak{U}^{i}$ and two threat point functions $T^{i} \in \mathfrak{T}^{i}$, with $i=1,2$, such that:

- the parameters $(y, \lambda)$ belong to $\mathcal{M}$ if and only if there exists a sharing $\left(\rho_{1}, \rho_{2}\right)$, with $\rho_{1}+\rho_{2}=y$, such that

$$
T^{i}(y, \lambda) \leq U^{i}\left(\rho_{i}, \lambda\right), \quad i=1,2,
$$

i.e., an agreement is reached if and only if the allocation $\left(T^{1}(y, \lambda)\right.$, $\left.T^{2}(y, \lambda)\right)$ lies within the Pareto frontier;

- in that case, the observed sharing $\left(\rho_{1}=\rho, \rho_{2}=y-\rho\right)$ solves:

$$
\max _{0 \leq \rho \leq y}\left(U^{1}(\rho, \lambda)-T^{1}(y, \lambda)\right) \cdot\left(U^{2}(y-\rho, \lambda)-T^{2}(y, \lambda)\right) .
$$

Note that, in the present set-up, Pareto-efficiency is an initial assumption that cannot be tested. Indeed, our goal is to focus on the other properties (independence of irrelevant alternatives, scale invariance and symmetry) that characterize the Nash solution. Regarding tests of Pareto efficiency (in a different but related context), the reader is referred to previous papers by Chiappori (1988, 1992) and Chiappori and Ekeland (2003, 2004).

\section{$2.2 \quad$ A negative result}

The answers to the two questions raised above - testability and identifiability - obviously depends on the prior information one is willing to exploit in the framework at stake. A first result is that the fully general setting, in which the form of utility and threat point functions is not restricted (except 
for Assumption S1-(a)), is simply too general. The answer to both questions is negative: Nash bargaining cannot generate testable predictions on observed outcomes, and the observation of the outcome does not allow to recover preferences. This is stated formally in the following Proposition.

Proposition 2 Let $\rho(y, \lambda)$ some function defined over $\mathcal{M}$, and whose range is included in $[0, y]$. Then, for any pair of utility functions $U^{1}, U^{2}$, satisfying Assumption S1-(a), there exist two threat point functions $T^{1}, T^{2}$ such that the agents' behavior is compatible with Nash bargaining.

Proof. For any given functions $U^{1}, U^{2}$, satisfying Assumption S1-(a), one can define $T^{1}, T^{2}$ by:

$$
\begin{array}{ll}
T^{i}(y, \lambda)=U^{i}\left(\rho_{i}(y, \lambda), \lambda\right) & \text { if }(y, \lambda) \in \mathcal{M}, \\
T^{i}(y, \lambda)>U^{i}(y, \lambda) & \text { if }(y, \lambda) \in \mathcal{N} .
\end{array}
$$

Then for any $(y, \lambda)$ in $\mathcal{N}$, no agreement can be reached, whereas for any $(y, \lambda)$ in $\mathcal{M}$, the sharing $\left(\rho_{1}(y, \lambda), \rho_{2}(y, \lambda)\right)$ is the only one compatible with individual rationality; thus it is obviously the Nash bargaining allocation.

The intuition of this result is straightforward: it is always possible to chose the status quo utilities $\left(T^{1}, T^{2}\right)$ equal to the agents' respective utilities at the observed outcome whenever an agreement is reached (so that, in practice, the chosen point is the only feasible point compatible with individual rationality), while making sure that $\left(T^{1}, T^{2}\right)$ is outside the Pareto frontier when agents are observed to disagree. Simple as it may seem, this argument still conveys two important messages. One is that when threat points are unknown, Nash bargaining has no empirical content (beyond Pareto efficiency); any efficient outcome can be reconciled with Nash bargaining. Secondly, the observation of the outcome brings no information on preferences (and in particular the concavity of the utility functions): any utilities can be made compatible with observed outcomes, using ad hoc threat points. Finally, it is important to stress that these negative results are by no means specific to Nash bargaining. The proof applies whatever the bargaining concept at stake, provided that it satisfies individual rationality — a very mild requirement indeed.

\subsection{Bargaining structure}

The negative result above does not mean that Nash bargaining (or, for that matter, bargaining theory altogether) cannot be tested, but simply that more 
structure is needed to achieve that goal. For example, the differentiability of $U^{i}$ and $T^{i}$ (such as required by Assumption S1-(b)) is sufficient to obtain some restrictions on the sharing function. However, our interpretation of testability is more demanding. We thus introduce the following 'non-parametric' assumptions on the bargaining structure.

Assumption S2 The threat points are independent of the size of the pie $y$; i.e., $\partial T^{i} / \partial y=0, i=1,2$.

Assumption S3 There exists a partition $\lambda=\left(\lambda_{1}, \lambda_{2}, \bar{\lambda}\right)$, with

$$
\lambda_{1}=\left(\lambda_{1}^{1}, \ldots, \lambda_{1}^{m}\right), \quad \lambda_{2}=\left(\lambda_{2}^{1}, \ldots, \lambda_{2}^{n}\right), \quad \bar{\lambda}=\left(\bar{\lambda}^{1}, \ldots, \bar{\lambda}^{l-m-n}\right)
$$

and $m \geq 1, n \geq 1, l \geq m+n$, such that neither $U^{i}$ nor $T^{i}$ depend on $\lambda_{j}$, where $i, j=1,2$ and $i \neq j$; i.e., $\partial U^{i} / \partial \lambda_{j}=\partial T^{i} / \partial \lambda_{j}=0$.

The additional structure given by these assumptions should a priori increase the empirical content of the bargaining game. Assumption S2 is standard; it is typical, for instance, of situations where, in the absence of an agreement, the opportunity at stake in the bargaining (the pie) is totally lost. Assumption S3 is an exclusion restriction that provides the key structure needed for testability. ${ }^{7}$ It states that for each player, there exists one parameter (at least) which does not affect the preferences of this player.

\section{Testability}

We now study the properties of the Nash bargaining model under Assumptions S1-S3. For the sake of presentation, we first leave aside the situations in which (a) the players disagree or (b) the players are indifferent between agreeing and disagreeing. Formally, we thus make the simplifying assumption (which will be relaxed in Section 5) that:

\footnotetext{
${ }^{7}$ Testable restrictions can be obtained without Assumption S2 provided that $\lambda_{1}$ and $\lambda_{2}$ are multi-dimensional vectors. For an investigation along those lines, see Chiappori and Donni (2005).
} 
Assumption $\mathbf{O 1}$ For any $(y, \lambda) \in \mathcal{S}$, there exists a sharing $\left(\rho_{1}, \rho_{2}\right)$, with $\rho_{1}+\rho_{2}=y$, such that $U^{i}\left(\rho_{i}, \lambda\right)-T^{i}(y, \lambda)>0$ for $i=1,2$.

Hence, an agreement is always reached, i.e., $\mathcal{S}=\mathcal{M}$. Moreover, we suppose that when an agreement is reached, each player receives a positive share of the pie. Formally:

Assumption $\mathbf{O} 2$ For any $(y, \lambda) \in \mathcal{M}$, the solution $\rho$ to Programme $\mathrm{P}$ is interior, i.e., $0<\rho_{i}(y, \lambda)<y$.

Then, under Assumptions S1-S3, the sharing function $\rho$ is defined as a function of $(y, \lambda)$ over the entire space $\mathcal{S}$, and solves the problem:

$$
\max _{0 \leq \rho \leq y}\left(U^{1}\left(\rho, \lambda_{1}, \bar{\lambda}\right)-T^{1}\left(\lambda_{1}, \bar{\lambda}\right)\right) \cdot\left(U^{2}\left(y-\rho, \lambda_{2}, \bar{\lambda}\right)-T^{2}\left(\lambda_{2}, \bar{\lambda}\right)\right) .
$$

The first order condition of this program is of the form:

$$
F^{1}\left(\rho, \lambda_{1}, \bar{\lambda}\right)=F^{2}\left(y-\rho, \lambda_{2}, \bar{\lambda}\right)
$$

with

$$
F^{i}\left(\rho_{i}, \lambda_{i}, \bar{\lambda}\right)=\frac{\partial U^{i} / \partial \rho_{i}}{U^{i}\left(\rho_{i}, \lambda_{i}, \bar{\lambda}\right)-T^{i}\left(\lambda_{i}, \bar{\lambda}\right)} .
$$

Our first result is that, under these additional specifications, the answer to the testability question is now positive. There exist strong testable restrictions on $\rho$ generated by the Nash-bargaining approach. Specifically, a first, simple result is the following:

Proposition 3 Under Assumptions S1-S2 and O1-O2, if the agents' behavior $(\{\mathcal{M}, \mathcal{N}\}, \rho)$ is compatible with Nash bargaining, then the function $\rho(y, \lambda)$ is twice continuously differentiable, with a range included in $] 0, y[$, and satisfies:

$$
0<\frac{\partial \rho}{\partial y}<1
$$

Proof. If equation (3) is differentiated with respect to $y$, one gets:

$$
-\frac{\partial F^{2}}{\partial \rho_{2}}+\left(\frac{\partial F^{1}}{\partial \rho_{1}}+\frac{\partial F^{2}}{\partial \rho_{2}}\right) \cdot \frac{\partial \rho}{\partial y}=0 .
$$


It is easily shown that $\partial F^{i} / \partial \rho_{i}<0$ for $i=1,2$ since the functions $U^{i}$ are strictly increasing and concave in $\rho_{i}$. Hence,

$$
\left.\frac{\partial \rho}{\partial y}=\frac{\partial F^{2} / \partial \rho_{2}}{\partial F^{1} / \partial \rho_{1}+\partial F^{2} / \partial \rho_{2}} \in\right] 0,1[,
$$

with $\partial F^{1} / \partial \rho_{1}+\partial F^{2} / \partial \rho_{2}<0$.

A Corollary of Proposition 3 is the following:

Corollary 4 The function $\rho$ can be globally inverted in $y$ on $\mathcal{S}$; i.e., there exists a function $\theta(\rho, \lambda)$, defined on the relevant space, such that

$$
\theta(\rho(y, \lambda), \lambda)=y \text { for all }(y, \lambda) \text { in } \mathcal{S} \text {. }
$$

Proof. The result is an immediate consequence of the implicit function theorem.

In words, $\theta(\rho, \lambda)$ is the size of the pie that will, for given $\lambda$, result in member 1 receiving a share of size $\rho$; from an empirical perspective, observing $\rho$ as a function of $(y, \lambda)$ is exactly equivalent to observing $y$ as a function $\theta$ of $(\rho, \lambda)$. It turns out that, from a theoretical viewpoint, many of the properties derived below can be expressed in a much simpler way using the function $\theta$.

As an illustration, one can derive a second and much stronger testable property of Nash-bargained sharing rules:

Proposition 5 Under Assumptions S1-S3 and O1-O2, if the agents' behavior $(\{\mathcal{M}, \mathcal{N}\}, \rho)$ is compatible with Nash bargaining, then the following, equivalent conditions hold:

- the function $\theta(\rho, \lambda)$ satisfies:

$$
\frac{\partial}{\partial \lambda_{2}^{t}}\left(\frac{\partial(\theta-\rho) / \partial \lambda_{1}^{s}}{\partial(\theta-\rho) / \partial \rho}\right)=0
$$

- the function $\rho(y, \lambda)$ satisfies:

$$
\frac{\partial \rho}{\partial \lambda_{1}^{s}}\left(\frac{\partial^{2} \rho}{\partial \lambda_{2}^{t} \partial y} \frac{\partial \rho}{\partial y}-\frac{\partial^{2} \rho}{\partial y^{2}} \frac{\partial \rho}{\partial \lambda_{2}^{t}}\right)+\left(1-\frac{\partial \rho}{\partial y}\right)\left(\frac{\partial^{2} \rho}{\partial \lambda_{1}^{s} \partial \lambda_{2}^{t}} \frac{\partial \rho}{\partial y}-\frac{\partial^{2} \rho}{\partial \lambda_{1}^{s} \partial y} \frac{\partial \rho}{\partial \lambda_{2}^{t}}\right)=0
$$

for all $s=1, \ldots, m$ and $t=1, \ldots, n$. 
Proof. Since $\partial F^{2} / \partial \rho_{2}<0$, equation (3) can be locally inverted as:

$$
y-\rho=\theta(\rho, \lambda)-\rho=\psi^{2}\left[F^{1}\left(\rho, \lambda_{1}, \bar{\lambda}\right), \lambda_{2}, \bar{\lambda}\right] .
$$

This condition implies that the function $\theta(\rho, \lambda)-\rho$ is separable:

$$
\frac{\partial(\theta-\rho) / \partial \lambda_{1}^{s}}{\partial(\theta-\rho) / \partial \rho}=\frac{\partial F^{1}\left(\rho, \lambda_{1}, \bar{\lambda}\right) / \partial \lambda_{1}^{s}}{\partial F^{1}\left(\rho, \lambda_{1}, \bar{\lambda}\right) / \partial \rho} \text { for all } s
$$

which immediately implies condition (6). Expressing this conditions using the function $\rho$ gives condition (7). Finally, one can readily check that a similar computation with $F^{2}$ instead of $F^{1}$ leads to the same equation.

In other words, Propositions 3 and 5 show that when the econometrician's information about the structure of the game is described by Assumptions 1-3, the Nash bargaining solution can be falsified (in Popper's terms) by observable behavior. Specifically, condition (5) states that any increase in the size of the pie must benefit both agents; it is a direct consequence of Assumption S2. On the other hand, conditions (6) or (7) translate the particular functional structure of equation (3) which defines the sharing function. Two remarks are in order at this point.

Remark 1. The conditions stated in Proposition 5 are not specific to the Nash solution. Indeed, from equation (3) one can see that any solution which can be obtained by the maximization of an index of the form:

$$
H\left(h^{1}\left(U^{1}\left(\rho, \lambda_{1}\right)-T^{1}\left(\lambda_{1}\right)\right)+h^{2}\left(U^{2}\left(y-\rho, \lambda_{2}\right)-T^{2}\left(\lambda_{2}\right)\right)\right),
$$

for some strictly increasing functions $h^{1}, h^{2}$ and $H$, will satisfy conditions (6) (or (7)). The concepts leading to such a maximization include, besides Nash, the Weighted Nash solution with constant bargaining weights, the Egalitarian solution and the Utilitarian solution; interestingly, these concepts all satisfy the independence of irrelevant alternatives (IIA) property. On the contrary, conditions (6) (or (7)) are not satisfied by the Kalai-Smorodinsky solution or the Yu solution, which do not satisfy the IIA property. ${ }^{8}$

\footnotetext{
${ }^{8}$ See Thomson (1994) for a taxonomy of bargaining solutions.
} 
Remark 2. Even when either the threat point $T^{i}$ or the bargaining surplus $U^{i}-T^{i}$ is monotonic in $\lambda_{i}$, it does not follow in general that $i$ 's share $\rho_{i}$ increases (or decreases) with $\lambda_{i}$. The reason for that is that since $U^{i}$ also depends on $\lambda$, changing $\lambda$ affects the marginal utility of income, which plays a key role in the determination of the solution. It can actually be shown that $\rho_{i}$ is increasing in $\lambda_{i}$ if and only if the marginal utility of income $\partial U^{i} / \partial \rho_{i}$ is more elastic with respect to $\lambda_{i}$ than the bargaining surplus $U^{i}-T^{i}$.

The conditions stated in Propositions 3 and 5 can be exploited to test whether players make use of the Nash solution. To do that, the simplest way is to translate conditions (5) and (6) (or (7)) into parameter constraints of a functional form. An illustration is provided by the following example.

Parametric example 1. For the sake of notational simplicity, we omit $\bar{\lambda}$ and assume that the vectors $\lambda_{i}$ are one dimensional. We then choose the following, 'semi-parametric' specification for the sharing function:

$$
\rho=y \cdot \mathcal{L}\left(a_{00}+a_{01} \lambda_{1}+a_{02} \lambda_{2}+a_{11} \lambda_{1}^{2}+a_{22} \lambda_{2}^{2}+a_{12} \lambda_{1} \lambda_{2}\right)
$$

where

$$
\mathcal{L}(x)=\frac{1}{1+\exp (x)}
$$

is the logistic distribution function; in words, the respective shares $\rho / y$ are taken to be logistic transformations of a general second order approximation. This form implies, as expected, that $\rho\left(y, \lambda_{1}, \lambda_{2}\right)$ is necessarily comprises between 0 and $y$. Moreover, condition (5) is globally satisfied and condition (6) requires that:

$$
a_{12}=0 .
$$

If this restriction is satisfied, the first order condition (3) gives:

$$
\rho \exp \left(a_{00}+a_{01} \lambda_{1}+a_{11} \lambda_{1}^{2}\right)=(y-\rho) \exp \left(-\left(a_{02} \lambda_{1}+a_{22} \lambda_{1}^{2}\right)\right) .
$$

Hence an econometric test of the Nash solution, under Assumptions S1-S3, boils down to testing that $a_{12}=0$. Finally, note that this example can be generalized with an approximation of any arbitrary order. 


\section{Identification}

\subsection{A non identifiability result}

We now consider the identification problem; i.e., we ask whether the utility and threat point functions can be retrieved from the observation of the sharing function. Since Nash bargaining is invariant by affine transformation of individual utilities, we say that utility functions $U$ and $U^{*}$ (resp. threat-point functions $T$ and $T^{*}$ ) are different if and only if there does not exist positive scalars $a$ and $b$ such that $U^{*}=a U+b\left(\right.$ resp. $\left.T^{*}=a T+b\right)$.

The main conclusion, then, is that the model is not identified; formally:

Proposition 6 Let $\rho(y, \lambda)$ be some twice continuously differentiable function defined over $\mathcal{S}$, that satisfies conditions (5) and (7), and whose range is included in $] 0, y[$. Then there exists a continuum of different utility functions $U^{1}, U^{2}$ and threat point functions $T^{1}, T^{2}$, such that Assumptions S1-S3 are satisfied and the agents' behavior is compatible with Nash bargaining.

Proof. This proof is in two steps. The (non-)identification of $F^{1}, F^{2}$ is first examined. The (non-)identification of $U^{1}, U^{2}, T^{1}, T^{2}$ then follows.

Part 1. Consider first the case of agent 1. If condition (6) is fulfilled, each ratio

$$
\frac{\partial(\theta-\rho) / \partial \lambda_{1}^{s}}{\partial(\theta-\rho) / \partial \rho}
$$

with $s=1, \ldots, m$, can be written as some function $\Phi^{s}$ of $\left(\lambda_{1}, \bar{\lambda}, \rho\right)$. Then, the function $F^{1}$ defined in equation (4) must satisfy:

$$
\frac{\partial F^{1} / \partial \lambda_{1}^{s}}{\partial F^{1} / \partial \rho}=\Phi^{s}\left(\rho, \lambda_{1}, \bar{\lambda}\right)
$$

with $\partial F^{1} / \partial \rho \neq 0$, where $\Phi^{s}$ is a known function. Let $\phi_{K}$ be a level curve of $F^{1}$; i.e.,

$$
F^{1}\left(\rho, \lambda_{1}, \bar{\lambda}\right)=K \Leftrightarrow \rho=\phi_{K}\left(\lambda_{1}, \bar{\lambda}\right) .
$$

The existence of $\phi_{K}$ is guaranteed by the implicit function theorem. The system of equations (10) becomes:

$$
\frac{\partial \phi_{K}\left(\lambda_{1}, \bar{\lambda}\right)}{\partial \lambda_{1}^{s}}=-\Phi^{s}\left(\phi_{K}, \lambda_{1}, \bar{\lambda}\right), \text { with } s=1, \ldots, m
$$


It can be shown, if one differentiates this system with respect to $\lambda_{1}$ and $\bar{\lambda}$, that cross-derivatives restrictions of the form:

$$
\frac{\partial^{2} \phi_{K}\left(\lambda_{1}, \bar{\lambda}\right)}{\partial \lambda_{1}^{s} \partial \lambda_{1}^{s^{\prime}}}=\frac{\partial^{2} \phi_{K}\left(\lambda_{1}, \bar{\lambda}\right)}{\partial \lambda_{1}^{s^{\prime}} \partial \lambda_{1}^{s}},
$$

are automatically satisfied. Hence, this system of partial differential equations has a solution for any initial value and the level curves can be recovered (up to a function of $\bar{\lambda}$ ) using equations (12). These are monotonic in the sense that $\phi_{K^{*}}\left(\lambda_{1}, \bar{\lambda}\right)>\phi_{K}\left(\lambda_{1}, \bar{\lambda}\right)$ if $K^{*}<K$. Clearly, from these curves and this relation of monotonicity, the function $F^{1}\left(\rho, \lambda_{1}, \bar{\lambda}\right)$ is defined up to a transform $G(\cdot, \bar{\lambda})$, increasing in its first argument. Symmetrically, the function $F^{2}\left(y-\rho, \lambda_{2}, \bar{\lambda}\right)$ for agent 2 is defined up to the same transform $G(\cdot, \bar{\lambda})$. That is, assume that some $\bar{F}^{1}\left(\rho, \lambda_{1}, \bar{\lambda}\right)$ and $\bar{F}^{2}\left(y-\rho, \lambda_{2}, \bar{\lambda}\right)$ satisfy equation (3), and define $\Gamma^{i}\left(\rho_{i}, \lambda_{i}, \bar{\lambda}\right)=G \circ \bar{F}^{i}\left(\rho_{i}, \lambda_{i}, \bar{\lambda}\right)$; then:

$$
\Gamma^{1}\left(\rho, \lambda_{1}, \bar{\lambda}\right)=\Gamma^{2}\left(y-\rho, \lambda_{2}, \bar{\lambda}\right) .
$$

Note, in particular, that $\partial \bar{F}^{i} / \partial \rho_{i}<0$ by construction.

Part 2. The arbitrary transform $G$ is the only indetermination on $F^{1}$ and $F^{2}$. Once the transform has been picked up, one can choose an arbitrary function $T^{i}\left(\lambda_{i}, \bar{\lambda}\right)$, and consider the equation in $U^{i}\left(\rho_{i}, \lambda_{i}, \bar{\lambda}\right)$ :

$$
\Gamma^{i}\left(\rho_{i}, \lambda_{i}, \bar{\lambda}\right)=\frac{\partial \log \left(U^{i}\left(\rho_{i}, \lambda_{i}, \bar{\lambda}\right)-T^{i}\left(\lambda_{i}, \bar{\lambda}\right)\right)}{\partial \rho_{i}}
$$

where $\Gamma^{i}=G \circ \bar{F}^{i}$ for some $G$. Defining the function $\Psi^{i}\left(\rho_{i}, \lambda_{i}, \bar{\lambda}\right)$ by

$$
\Psi^{i}\left(\rho_{i}, \lambda_{i}, \bar{\lambda}\right)=\int_{1}^{\rho_{i}} \Gamma^{i}\left(\xi, \lambda_{i}, \bar{\lambda}\right) \mathrm{d} \xi
$$

the general solution to equation (13) is of the form:

$$
U^{i}\left(\rho_{i}, \lambda_{i}, \bar{\lambda}\right)=K^{i}\left(\lambda_{i}, \bar{\lambda}\right) \exp \Psi^{i}\left(\rho_{i}, \lambda_{i}, \bar{\lambda}\right)+T^{i}\left(\lambda_{i}, \bar{\lambda}\right)
$$

for an arbitrary, positive function $K^{i}\left(\lambda_{i}, \bar{\lambda}\right)$, where $\partial \Psi^{i} / \partial \rho_{i}=\Gamma^{i}(x, z)$. The constant $K^{i}\left(\lambda_{i}, \bar{\lambda}\right)$ does not affect the concavity of utility with respect to income, i.e., the condition above identifies a cardinal representation of the utility function (as a function of the share). Now, note that:

$$
\frac{\partial U^{i}}{\partial \rho_{i}}=K^{i}\left(\lambda_{i}, \bar{\lambda}\right) \exp \Psi^{i}\left(\rho_{i}, \lambda_{i}, \bar{\lambda}\right) \times \Gamma^{i}\left(\rho_{i}, \lambda_{i}, \bar{\lambda}\right)
$$


One can assume that $\Gamma^{i}>0$ over the domain at stake (it suffices to define $\left.\Gamma^{i}=\exp \bar{F}^{i}\right)$ so that the expression above is positive. Finally,

$$
\frac{\partial^{2} U^{i}}{\partial \rho_{i} \partial \rho_{i}}=K^{i}\left(\lambda_{i}, \bar{\lambda}\right) \exp \Psi^{i}\left(\rho_{i}, \lambda_{i}, \bar{\lambda}\right) \times\left[\frac{\partial \Gamma^{i}}{\partial \rho_{i}}+\left(\Gamma^{i}\left(\rho_{i}, \lambda_{i}, \bar{\lambda}\right)\right)^{2}\right] .
$$

The selected $G$ must be such that the term into brackets is negative. Since $\mathcal{S}$ is bounded, that can be obtained by the transform $\Gamma^{i}=k \exp \bar{F}^{i}$ where $k>0$ is an arbitrary small constant.

A by-product of the proof is that the conditions stated in Proposition 3 and 5 are sufficient as well, in that any sharing rule satisfying these conditions can be rationalized as a Nash bargaining solution for well-chosen utilities and threat points satisfying Assumptions S1-S3. However, the framework falls short of providing a uniqueness result; identification requires still more information. The intuition of this result is that, at best, the functions $F^{1}$ and $F^{2}$ in expression (3) are defined up to some (common) mapping $G$.

Parametric example 2. Coming back to our numerical example, with the semi-parametric specification for the sharing function:

$$
\rho=y \cdot \mathcal{L}\left(a_{00}+a_{01} \lambda_{1}+a_{02} \lambda_{2}+a_{11} \lambda_{1}^{2}+a_{22} \lambda_{2}^{2}\right) .
$$

Let

$$
\begin{aligned}
& g_{1}\left(\lambda_{1}\right)=\exp \left(a_{00}+a_{01} \lambda_{1}+a_{11} \lambda_{1}^{2}\right), \\
& g_{2}\left(\lambda_{2}\right)=\exp \left(-\left(a_{2} \lambda_{02}+a_{22} \lambda_{2}^{2}\right)\right) .
\end{aligned}
$$

Then, one can see that the functions $F^{i}$ are given by:

$$
\begin{aligned}
& F^{1}\left(\rho_{1}, \lambda_{1}\right)=G\left(\rho_{1} g_{1}\right), \\
& F^{2}\left(\rho_{2}, \lambda_{2}\right)=G\left(\rho_{2} g_{2}\right),
\end{aligned}
$$

where $G$ is an arbitrary function. For any choice of $G$, one can recover the utility functions for arbitrary choices of the threat points. For instance, for $G(x)=x$, we have that:

$$
\begin{aligned}
& U^{1}\left(\rho_{1}, \lambda_{1}\right)=K^{1}\left(\lambda_{1}\right) \exp \left(\frac{1}{2} g_{1} \rho_{1}^{2}\right)+T^{1}\left(\lambda_{1}\right), \\
& U^{2}\left(\rho_{2}, \lambda_{2}\right)=K^{2}\left(\lambda_{2}\right) \exp \left(\frac{1}{2} g_{2} \rho_{2}^{2}\right)+T^{2}\left(\lambda_{2}\right) .
\end{aligned}
$$


where $K^{1}\left(\lambda_{1}\right)$ and $K^{2}\left(\lambda_{2}\right)$ are some positive functions. However, this transform is not convenient because the resulting utility functions are not concave. For $G(x)=x^{-1}$, we have that:

$$
\begin{aligned}
& U^{1}\left(\rho_{1}, \lambda_{1}\right)=K^{1}\left(\lambda_{1}\right) \rho_{1}^{1 / g_{1}}+T^{1}\left(\lambda_{1}\right), \\
& U^{2}\left(\rho_{2}, \lambda_{2}\right)=K^{2}\left(\lambda_{2}\right) \rho_{2}^{1 / g_{2}}+T^{2}\left(\lambda_{2}\right) .
\end{aligned}
$$

Then, these expressions correspond to CRRA utility functions if $g_{1}, g_{2}>1$.

We now provide two examples of additional assumptions that enable to recover the underlying structural model from observed behavior.

\subsection{Case 1: one affine utility function}

A first solution is to assume that the utility of one agent (say, agent 2) is an affine function of the agent's share of the pie. Formally:

Assumption S4. There exists functions $\alpha$ and $\beta>0$ of $\left(\lambda_{2}, \bar{\lambda}\right)$ such that the agent 2's utility function can be written as $U^{2}=\alpha+\beta \cdot(y-\rho)$.

This may be the case, for instance, if agent 2 represents a risk-neutral employer who bargains with a trade-union. If so, the Nash program becomes:

$$
\max _{0 \leq \rho \leq y} \beta\left(\lambda_{2}, \bar{\lambda}\right)\left(U^{1}\left(\rho, \lambda_{1}, \bar{\lambda}\right)-T^{1}\left(\lambda_{1}, \bar{\lambda}\right)\right) \cdot\left((y-\rho)-\gamma\left(\lambda_{2}, \bar{\lambda}\right)\right),
$$

where $\gamma=\left(T^{2}-\alpha\right) / \beta$, showing that only the ratio $\gamma$ is relevant in the maximization programme. Hence $\alpha$ and $\beta$ cannot be identified. Equation (3) becomes:

$$
\frac{\partial U^{1} / \partial \rho_{1}}{U^{1}\left(\rho, \lambda_{1}, \bar{\lambda}\right)-T^{1}\left(\lambda_{1}, \bar{\lambda}\right)}=\frac{1}{(y-\rho)-\gamma\left(\lambda_{2}, \bar{\lambda}\right)}
$$

In this case, the sharing function has to satisfy additional restrictions. To see why, note that the right-hand side of equation (15) should only depend on $\rho, \bar{\lambda}$ and $\lambda_{1}$. Using the function $\theta$ introduced in Corollary 4, we have that:

$$
\frac{\partial}{\partial \lambda_{2}}\left(\theta\left(\rho, \lambda_{1}, \lambda_{2}, \bar{\lambda}\right)-\rho-\gamma\left(\lambda_{2}, \bar{\lambda}\right)\right)=0 .
$$

Hence

$$
\frac{\partial \theta}{\partial \lambda_{2}}\left(\rho, \lambda_{1}, \lambda_{2}, \bar{\lambda}\right)=\frac{\partial \gamma}{\partial \lambda_{2}}\left(\lambda_{2}, \bar{\lambda}\right)
$$


This equation pins down $\gamma$ up to some additive function of $\bar{\lambda}$. More importantly, it implies that

$$
\frac{\partial^{2} \theta}{\partial \lambda_{1} \partial \lambda_{2}}=\frac{\partial^{2} \theta}{\partial \rho \partial \lambda_{2}}=0
$$

which further restricts the sharing function.

The most important result is stated in the following Proposition.

Proposition 7 Under Assumptions S1-S4 and O1-O2, the knowledge of the sharing $\rho_{i}(\lambda, y)$ identifies player 1's utility function $U^{1}$ up to an affine, increasing transform, whose the coefficients are functions of $\lambda_{1}$ and $\bar{\lambda}$. In particular, the cardinal representation of the utility of player 1 is exactly identified.

Proof. One has previously shown, in the Proof of Proposition 6 (Part 1), that the functions $F^{1}$ and $F^{2}$ are defined up to the same transform $G(\cdot, \bar{\lambda})$, increasing in its first argument. However, in the present context, the assumption on $U^{2}$ limits the set of such transforms to linear transforms. More precisely, the function $G$ is defined by:

$$
G\left(\bar{F}^{1}\left(\lambda_{1}, \bar{\lambda}, \rho\right), \bar{\lambda}\right)=\frac{1}{(y-\rho)-\gamma\left(\lambda_{2}, \bar{\lambda}\right)}
$$

where $\bar{F}^{1}$ is a known function. Thus,

$$
\frac{-1}{G^{2}} \frac{\partial G}{\partial F^{1}}=\frac{(1-\partial \rho / \partial y)}{\partial \rho / \partial y}\left(\frac{\partial \bar{F}^{1}}{\partial \rho}\right)^{-1},
$$

which identifies $G$ up to a function of $\bar{\lambda}$. Then, using the same argument as in the Proof of Proposition 6 (Part 2), the function $U^{1}$ can be retrieved up to an affine transformation, whose the coefficients depend on $\lambda_{1}$ and $\bar{\lambda}$.

\subsection{Case 2: $\lambda$-independent utility functions}

The second particular case is a straightforward generalization of the experiment described in Introduction, where $\lambda_{1}$ and $\lambda_{2}$ were interpreted as the agents' reservation payments. The specific feature we shall keep from the experiment is that $\lambda_{1}$ and $\lambda_{2}$ are only relevant for the threat points; they have no direct impact on utilities. We now proceed to show that, in this context, not only additional restrictions are generated on the shape of the 
sharing function, but both individual utilities and threat points are uniquely recovered (up to the same affine transform).

Formally, we thus introduce the following assumption:

Assumption $\mathbf{S 4} \quad$ The individual utilities are independent of the parameters $\lambda_{1}$ and $\lambda_{2}$, i.e., $\partial U^{i} / \partial \lambda_{i}=0, i=1,2$. The vectors of parameters $\lambda_{1}$ and $\lambda_{2}$ are one dimensional and there is no vector of parameters $\bar{\lambda}$.

The assumption on the dimensionality of $\lambda_{1}$ and $\lambda_{2}$ is made for notational convenience and the parameters $\bar{\lambda}$ are omitted for the same reason. This could be relaxed. Then, under Assumptions S1-S3, S4', the sharing function $\rho\left(y, \lambda_{1}, \lambda_{2}\right)$ thus solves the problem:

$$
\max _{0 \leq \rho \leq y}\left(U^{1}(\rho, \bar{\lambda})-T^{1}\left(\lambda_{1}, \bar{\lambda}\right)\right) \cdot\left(U^{2}(y-\rho, \bar{\lambda})-T^{2}\left(\lambda_{2}, \bar{\lambda}\right)\right) .
$$

We can now state the main result:

Proposition 8 Assume $U^{i}$ is not exponential (i.e., $U^{i}\left(\rho_{i}\right)$ is not of the form $\alpha e^{\mu \rho_{i}}+\beta$ for some $\left.\alpha, \beta, \mu\right)$. Then, under Assumptions $\mathrm{S} 1-\mathrm{S} 3, \mathrm{~S}^{\prime}$ and $\mathrm{O} 1-$ $\mathrm{O} 2$, the knowledge of the sharing $\rho_{i}(y, \lambda)$ identifies $U^{i}$ and $T^{i}$ up to an affine, increasing transform.

The proof relies on the following Lemma:

Lemma 9 Let $F(x, y)$ be a given function, and assume that for some functions $B, C, G$ the following equation is satisfied:

$$
G(F(x, y))=\frac{B^{\prime}(x)}{B(x)-C(y)}
$$

Assume that $F$ is such that $\partial F(x, y) / \partial x \neq 0, \partial F(x, y) / \partial y \neq 0$, and $B(x)$ is not exponential. Then $B$ and $C$ are identified from $F$ up to the same affine transform.

Proof. The proof of the Lemma is in Appendix. We now show that the Lemma implies Proposition 8. As before, define $F^{1}$ and $F^{2}$ by

$$
F^{i}\left(\rho_{i}, \lambda_{i}\right)=\frac{\partial U^{i} / \partial \rho_{i}}{U^{i}\left(\rho_{i}\right)-T^{i}\left(\lambda_{i}\right)} .
$$


From the Proof of Proposition 6 (Part 1), we know that $F^{i}$ is identified up to some increasing transform; i.e., there exists some known function $\bar{F}^{i}$ such that:

$$
G\left(\bar{F}^{i}\left(\rho_{i}, \lambda_{i}\right)\right)=F^{i}\left(\rho_{i}, \lambda_{i}\right)=\frac{\partial U^{i} / \partial \rho_{i}}{U^{i}\left(\rho_{i}\right)-T^{i}\left(\lambda_{i}\right)}
$$

for some $G$. It remains to be shown that given the particular form at stake, the knowledge of $F^{i}$ up to an increasing transform is sufficient to identify $U^{i}$ and $T^{i}$. Clearly, Lemma 9 immediately implies the conclusion. Moreover, if $B$ and $C$ are identified up to the same affine transform, then $G(F(x, y))$ is exactly identified, hence $G$ as well.

Again, additional testable restrictions are generated by this particular form. These conditions are technical, and we omit them in the present paper. It can be demonstrated, however, that the logistic-quadratic form used in the empirical example above is not compatible with this setting. In other words, an empirical model of bargaining that is using the logistic-quadratic specification must assume (at least implicitly) that individual utilities in case of an agreement depend on the threat point payment - a strong assumption indeed. This remark illustrates the relevance of a preliminary, theoretical investigation. An empirical specification based on the logistic-quadratic form may be quite appealing (and fit the data); but it is internally inconsistent with the model at stake, at least if one assumes (as it seems natural) that agents care about their threat point utility only insofar as it affects the bargaining outcome.

\section{The agreement frontier}

In the previous Section, it is assumed that cooperation always generates a positive surplus that can be shared between the players. From now on, we consider a more general case: $\mathcal{M} \subseteq \mathcal{S}$ so that the possibility of a disagreement between the players, or an agreement along the boundary of $\mathcal{M}$, can no longer be excluded.

To begin with, it is worth noting that, when $(y, \lambda) \in \mathcal{N}$, i.e., the players do not agree about the sharing of the pie, the outside econometrician can learn next to nothing about the underlying structure of the bargaining. In particular, the utility functions cannot be identified. The econometrician can only infers from the observation of the disagreement that, whatever the 
sharing of the pie may be, and for one player at least, the utility obtained from the reservation payment must be higher than what is obtained from the share of the pie.

However, the study of the agreement frontier - the locus where the players are indifferent whether the agreement is reached or not - is much more interesting. Indeed, along this frontier, the econometrician observes the sharing of the pie, as a function of the size of the pie and the set of parameters, and knows that, by definition, the bargaining surplus is exactly equal to zero. Formally, the agreement frontier is defined as follows:

Definition 10 The agreement frontier $\mathcal{F}$ is the subset of $(y, \lambda) \in \mathcal{S}$ such that each agent is indifferent between her share of the pie and her reservation payment, i.e.,

$$
\begin{aligned}
\mathcal{F}= & \left\{(y, \lambda) \in \mathcal{S} \text { such that } U^{1}(\rho, \lambda)=T^{1}(\lambda), U^{2}(y-\rho, \lambda)=T^{2}(\lambda),\right. \\
& \text { for some } \rho, \text { with } 0 \leq r \leq y\} .
\end{aligned}
$$

The observable agreement frontier $\mathcal{F}^{*}$ is a subset of $\mathcal{F}$ defined by $\mathcal{M} \cap \operatorname{cl}(\mathcal{N})$.

A first, trivial result is that the knowledge of the threat points uniquely defines utilities (along the observable frontier). More importantly, the agreement frontier, which generated by the structure of the bargaining, should have some features that can be tested. Before examining that, we introduce the following assumption that is used throughout this Section.

Assumption 03 There is some $(y, \lambda) \in \mathcal{S}$ such that each agent is indifferent between her share of the pie and her reservation payment, i.e., $\mathcal{F} \neq \varnothing$.

The following Proposition presents a set of testable restrictions which are based on the observation of the sole agreement frontier.

Proposition 11 Under Assumptions S1-S2 and O3, if the agents' behavior $(\{\mathcal{M}, \mathcal{N}\}, \rho)$ is compatible with Nash bargaining, there exists a subset $\mathcal{B}$ in $\mathbb{R}^{l}$, and a three times continuously differentiable function $\sigma(\lambda)$ defined over $\mathcal{B}$, such that $y=\sigma(\lambda)$ if and only if $(y, \lambda) \in \mathcal{F}$, and

(i) if $(y, \lambda) \in \mathcal{M}$ and $\lambda \in \mathcal{B}$, then $y \geq \sigma(\lambda)$,

(ii) if $(y, \lambda) \in \mathcal{N}$ and $\lambda \in \mathcal{B}$, then $y<\sigma(\lambda)$.

Moreover, under Assumption S3, the function $\sigma(\lambda)$ is additive in the sense that $\sigma(\lambda)=\sigma_{1}\left(\lambda_{1}, \bar{\lambda}\right)+\sigma_{2}\left(\lambda_{2}, \bar{\lambda}\right)$ for some functions $\sigma_{1}\left(\lambda_{1}, \bar{\lambda}\right)$ and $\sigma_{2}\left(\lambda_{2}, \bar{\lambda}\right)$. 
Proof. Consider the system of equations,

$$
\begin{aligned}
U^{1}\left(\rho, \lambda_{1}, \bar{\lambda}\right)-T^{1}\left(\lambda_{1}, \bar{\lambda}\right) & =0, \\
U^{2}\left(y-\rho, \lambda_{2}, \bar{\lambda}\right)-T^{2}\left(\lambda_{2}, \bar{\lambda}\right) & =0,
\end{aligned}
$$

which implicitly defines $\rho$ and $y$ as a function of $\lambda$. Inverting (17) with respect to $\rho$ yields:

$$
\rho=\sigma^{1}\left(\lambda_{1}, \bar{\lambda}\right) .
$$

Hence, the sharing function is independent of $\lambda_{2}$ and $y$ along the agreement frontier. Similarly, inverting (18) with respect to $y-\rho$ yields:

$$
y-\rho=\sigma^{2}\left(\lambda_{2}, \bar{\lambda}\right) .
$$

Then, substituting equation (19) into equation (20) proves that $\sigma(\lambda)$ is additive in the sense of Proposition 11.

In other words, the first part of the Proposition states that the agreement frontier can be equivalently written as:

$$
y=\sigma(\lambda) .
$$

The players will not agree about the sharing of a pie whose the size is less than a reservation value, given by $\sigma(\lambda)$; and an agreement will occur if the size of the pie exceeds its reservation value. Note that, formally, $y<\sigma(\lambda)$ does not imply that $(y, \lambda) \in \mathcal{N}$ because the reservation value of $y$ may well be outside of $\mathcal{S}$. The second part of the Proposition yields a very strong, testable restriction on the form of the agreement frontier.

Since the results in Proposition 11 are based on the sole observation of the agreement frontier, the econometrician can make a test of Nash bargaining without observing the sharing of the pie (at least if $\mathcal{F}^{*} \neq \varnothing$ ). However, more can be obtained if the sharing of the pie is observed. This is formally stated in the following Proposition:

Proposition 12 Under Assumptions S1-S3 and O2-O3, if the agents' behavior $(\{\mathcal{M}, \mathcal{N}\}, \rho)$ is compatible with Nash bargaining, then:

$$
\frac{\partial \sigma}{\partial \lambda_{1}^{s}}=\frac{\partial \rho / \partial \lambda_{1}^{s}}{(1-\partial \rho / \partial y)}, \quad \frac{\partial \sigma}{\partial \lambda_{2}^{t}}=-\frac{\partial \rho / \partial \lambda_{2}^{t}}{\partial \rho / \partial y}, \quad \text { for any }(y, \lambda) \text { in } \mathcal{F}
$$

and for any $s=1, \ldots, m$ and any $t=1, \ldots, n$. 
Proof. As a direct consequence of equations (19), one has:

$$
\sigma_{1}\left(\lambda_{1}, \bar{\lambda}\right)=\rho\left(\sigma_{1}\left(\lambda_{1}, \bar{\lambda}\right)+\sigma_{2}\left(\lambda_{2}, \bar{\lambda}\right), \lambda_{1}, \lambda_{2}, \bar{\lambda}\right) .
$$

Differentiating this expression with respect to $\lambda_{1}^{s}$ and $\lambda_{2}^{t}$ gives the conditions in Proposition 12

Finally, note that these conditions are not sufficient. Indeed, as previously shown, the functions $F^{i}\left(\rho_{i}, \lambda_{i}, \bar{\lambda}\right)$ for $i=1,2$ are defined up to some increasing function $G$. Remember now that

$$
F^{i}\left(\rho_{i}, \lambda_{i}, \bar{\lambda}\right)=\frac{\partial U^{i} / \partial \rho_{i}}{U^{i}\left(\rho_{i}, \lambda_{i}, \bar{\lambda}\right)-T^{i}\left(\lambda_{i}, \bar{\lambda}\right)} .
$$

Hence, any particular solution $\bar{F}^{i}\left(\rho_{i}, \lambda_{i}, \bar{\lambda}\right)$ has to satisfy a boundary condition, i.e., $\lim _{y \rightarrow \gamma} \bar{F}^{i}\left(\rho_{i}(y, \lambda), \lambda_{i}, \bar{\lambda}\right)=\infty$.

\section{Applications}

Our main results leads to a significant qualification of the widely accepted views that "bargaining theory contains very few interesting propositions that can be tested empirically", to quote Hamermesh (1973, p. 1146). Admittedly, testability and identifiability do not obtain in the most general model. If the econometrician knows nothing about the form of utility and threat point functions, any sharing of the pie is compatible with Nash bargaining. Nevertheless, whenever utility and threat point functions satisfy some specific exclusion property, Nash bargaining generates a set of strong restrictions on observed behavior. An analogy with consumer theory is helpful at that point. As is well-known, a system of demands must satisfy a set of testable restrictions (homogeneity, symmetry, negativity). Still the maximization of utility functions has an empirical content only if utility functions are, quite naturally, assumed to be independent of prices and incomes. A similar condition of exclusion is required in the Nash bargaining context. The pertinence of this condition cannot be judged a priori but depends on the bargaining context.

Our results should be considered as a first step towards a better understanding of the empirical content of bargaining theory. Potential applications are numerous. A non-exhaustive list of examples is given below. 
Trade-union negotiations The objectives of the union and those of the firms are to some extent in opposition to each other, and the observed outcomes will not in general be precisely the most preferred outcome of either party. This conflict is often solved by assuming a Nash solution. Still the latter has to be supported by empirical arguments. Hence there has been several attempts to test the Nash solution in the sense of seeing if actual negotiated agreements are consistent with the Nash model (see De Menil (1971), Hamermesh (1973), Sevjnar (1980, 1986), Coles and Hildreth (2000) for instance). However, these tests are relatively crude. The objective of the firm is simply assumed to maximize profit. The union is usually assumed to be a rent-maximizer or to have a linear utility function. The message of our paper is that, even without assumptions on the parametric form of utility functions, Nash solutions have a strong empirical content. This sheds a new light on this classical field of research.

Of particular interest, in this context, is Proposition 7. Indeed, in many cases the linearity assumption makes sense on the firm's side. Profit maximization is a standard theoretical assumption, and risk neutrality can be derived from specific assumptions on, say, financial markets. The same assumption, however, is more debatable when made for the workers. Our results show that the latter assumption is by no means necessary. Not only can Nash bargaining be tested without this assumption, but the union's preferences (and in particular the degree of concavity of their utility) can in principle be identified from the outcome of the negotiation.

Household behavior During the last two decades, several models of household behavior accounting for the fact that spouses' goals may differ have emerged. Specifically, Chiappori (1988a, 1992) relies on the sole assumption that the intrahousehold decision process is efficient, while Manser and Brown (1980), McElroy and Horney (1981) and Lundberg and Pollak (1993) refers to some cooperative equilibrium concept (typically Nash bargaining). Since Nash-bargaining generates efficient outcomes, the second approach is a particular case of the first. An interesting problem is whether (and under which conditions) the additional structure provided by Nash-bargaining results in either additional testable predictions on behavior, or a more accurate identification of individual preferences and decision processes. ${ }^{9}$

\footnotetext{
${ }^{9}$ See Chiappori $(1988 b, 1991)$ and McElroy and Horney (1990) for an exchange on this issue.
} 
Our results suggest that the answer depends on the level of structure one is willing to introduce into the model. Proposition 2 implies that the Nash bargaining assumption, per se, implies very little beyond efficiency a conclusion already conjectured by Chiappori (1991). More surprisingly, however, Proposition 5 suggests that mild assumptions may be sufficient to reverse this conclusion. For instance, in a model with purely private consumption, in which the decision process can be decentralized, the form of the so-called sharing rule may indeed be constrained by the Nash bargaining context, even when the threat points are not explicitly specified. ${ }^{10}$

Experimental economics The investigation of bargaining theory in experimental economics dates back to the seminal works by Siegel and Fouraker (1960). A standard problem with experiments of this type is that the observer does not know the players' preferences. As we said in Introduction, assuming linear preferences may unduly restrict the scope of the test: a joint test of Nash bargaining and linear preferences is likely to be rejected just because preferences fail to be linear - and then the rejection tells very little about the status of the Nash bargaining hypothesis.

A possible solution, introduced by Roth and Malouf (1979), is to consider players who bargain about probabilities of a lottery. The idea, here, is that linearity immediately follows from the expected utility hypothesis. Note, however, that once again one jointly tests Nash bargaining and expected utility. Given that expected utility tends to be rejected in experiments, once again the status of the test (as a test of Nash bargaining) is ambiguous at best.

From this point of view, the methodology developed in this paper opens new and interesting directions for future research in this area. Consider again the simple experiment discussed in Introduction. Our main conclusion is that a cardinal representation of each agent's utility function can be identified from it. This identification does not require any form of uncertainty; in particular, it does not rely on the assumption that utilities are of VNM type. Moreover, the Nash bargaining structure generates strong testable properties for the sharing function.

The possibility of identifying a cardinal representation of individual utilities in the absence of uncertainty raises interesting perspectives. The mere

\footnotetext{
${ }^{10}$ The application of our results to the context of household behavior raises however specific problems. The interested reader is referred to Chiappori and Donni (2004).
} 
fact that Nash bargaining involves cardinal representations of individual utilities (i.e., concavity matters) even in the absence of uncertainty can be given various interpretations. One of these relies on the non-cooperative foundations of Nash bargaining, which do involve randomness. ${ }^{11}$ While interesting, this interpretation raises however several problems. First, the noncooperative interpretation provided by Binmore, Rubinstein and Wolinsky relies on expected utility. This requirement is somewhat problematic: it is hard to see why the use of Nash bargaining should be restricted to preferences compatible with expected utility maximization, rather than more general preferences under uncertainty. Recent progress have been made in this direction by Rubinstein, Safra and Thomson (1992), who extend the interpretation of Nash-bargaining to a family of non-expected utility preferences. Still, why the definition of Nash bargaining should rely at all on preferences on lotteries is not clear. After all, non cooperative models are not the only justification of Nash bargaining, and possibly not the most convincing one. The initial definition of Nash bargaining was axiomatic; and none of the axioms used by Nash in his original contribution did rely on decision under uncertainty in any manner. Moreover, Nash bargaining is used in a variety of situations, most of which involve no uncertainty. ${ }^{12}$

In other words, the interpretation just described, based on the idea that concavity of the utility function matters in Nash bargaining because Nash bargaining should be viewed as a reduced form for some non cooperative game that does involve randomness, needs not be the ultimate one. It should in particular be put in perspective with a standard claim made by (some) tenants of non expected utility approaches, and stating that concavity of utility has little to do with risk aversion. Decreasing marginal utility of income, it is argued, relates to psychological patterns of individual satisfaction that can be understood independently of any risk. Risk aversion, in this perspective, is a completely different issue, which is (at least in some versions) related to transformations of the probability distribution.

The theoretical debate is both stimulating, challenging and intricate. However, a very interesting question is whether there could be an empiri-

\footnotetext{
${ }^{11}$ See Binmore, Rubinstein and Wolinsky(1986), and Myerson (1990, Chapter 8) for a very pedagogical presentation.

${ }^{12}$ Moreover, even in situations where the bargaining game is indeed non cooperative and involves uncertainty, the game at stake may fail to fit the formal structure referred to by the Binmore-Rubinstein-Wolinsky framework (alternative offers, exogenous termination probabilities, etc.)
} 
cal answer to the debate. In other words, could there be a way of directly testing the relationship between decreasing marginal utility of income and risk aversion? The obvious problem with such a program is that the decreasingness of marginal utility of income is hard (or impossible, it is often argued) to assess in a context of certainty. Our suggestion is that Nash bargaining may actually provide such an assessment. We believe, in other words, that it may be worth trying to take the theory literally and trying to recover the concavity of individual utilities from the observation of negotiations between agents in the absence of uncertainty. From this perspective, the tools provided by this paper may be useful precisely because they show how individual utilities can be retrieved (up to an affine transform) in a bargaining context.

Whether the level of concavity implicit in the Nash bargaining outcome is correlated with the individuals' attitude toward risk is an interesting empirical question. After all, the same person may in principle be a tough negotiator and a risk averse decision maker. At any rate, an experiment should be easy to perform. It should go along the following lines:

1. face each individual of a given group with a standard choice between lotteries, in order to assess her level of risk aversion

2. match randomly the agents by pairs, and let them play a two-sided bargaining problem identical to the one discussed in Introduction; use the theoretical approach described in this paper to recover their utility functions

3. compare the two sets of results. According to the standard interpretation, more risk averse individuals, being characterized by more concave VNM utilities, should perform poorly in the bargaining stage; an empirical check of this prediction would be quite illuminating.

4. Interestingly enough, this approach has various by-products. For instance, the idea that risk aversion has more to do with probability transformation than with decreasing marginal utility of consumption could be taken to data in a systematic way: if one believes that the concavity retrieved from the second phase has general relevance, then it can be plugged into the first stage to recover possible probability transformations.

Experiments of this kind will be the topic of future work. 


\section{A Appendix : Proof of Lemma 9}

In this appendix, the notation $f_{x}$ stands for the differential of function $f$ with respect to variable $x$; the notation $f^{\prime}$ is used when $f$ has only one argument.

Note first that $G(F(x, y))$ is of the form:

$$
G(F(x, y))=\frac{A(x)}{B(x)-C(y)},
$$

where $A(x)=B^{\prime}(x)$. It follows that

$$
\frac{F_{x}(x, y)}{F_{y}(x, y)}=\frac{A^{\prime}(x)(B(x)-C(y))-A(x) B^{\prime}(x)}{A(x) C^{\prime}(y)} .
$$

Define:

$$
\phi(x, y)=\frac{F_{x}(x, y)}{F_{y}(x, y)} .
$$

Note that $\phi$ is a known function, i.e., it does not depend on $G$, and is such that

$$
\log \phi(x, y)=\log \left(\frac{\partial F(x, y)}{\partial x}\right)-\log \left(\frac{\partial F(x, y)}{\partial y}\right)
$$

Consider equation (21) as an equation in $A=B^{\prime}, B, C$. We now show that generically on $\phi$, this equation identifies $B, C$ up to an affine transform. We now distinguish two cases, depending on whether $\log \phi(x, y)=$ $\log \left(F_{x}(x, y)\right)-\log \left(F_{y}(x, y)\right)$ is additively separable in $x$ and $y$ or not.

CASE 1 (GENERAL CASE): $\log \phi(x, y)$ is not additively separable in $x$ and $y$. The proof goes in 3 steps

Step 1: Define $v(x)=A^{\prime}(x) / A(x)$ and $w(x)=v(x) B(x)-B^{\prime}(x)$, then equation (21) becomes:

$$
\phi(x, y) C^{\prime}(y)+v(x) C(y)=w(x) .
$$

Differentiating with respect to $x$ yields:

$$
\phi_{x}(x, y) C^{\prime}(y)+v^{\prime}(x) C(y)=w^{\prime}(x) .
$$

If

$$
\phi_{x}(x, y) v(x)-\phi(x, y) v^{\prime}(x)=0,
$$


then $\phi(x, y)=D(y) v(x)$ for some function $D$ and $\log \phi(x, y)$ is additively separable in $x$ and $y$ which contradicts the assumption. Hence the expression is non zero, and from equations (22) and (23) one gets:

$$
\begin{aligned}
C(y) & =\frac{\phi_{x}(x, y) w(x)-\phi(x, y) w^{\prime}(x)}{\phi_{x}(x, y) v(x)-\phi(x, y) v^{\prime}(x)} \\
C^{\prime}(y) & =\frac{v(x) w^{\prime}(x)-v^{\prime}(x) w(x)}{\phi_{x}(x, y) v(x)-\phi(x, y) v^{\prime}(x)} .
\end{aligned}
$$

A first necessary condition expresses the fact that the derivative of the righthand-side of equation (24) equals the right-hand-side of equation (25). This gives either

$$
\begin{aligned}
w^{\prime}(x) v(x)-v^{\prime}(x) w(x) & =0 \\
\phi_{x}(x, y) v(x)+\phi_{y}(x, y) \phi_{x}(x, y)-\phi_{x y}(x, y) \phi(x, y)-\phi(x, y) v^{\prime}(x) & =0 .
\end{aligned}
$$

If $\left(w^{\prime}(x) v(x)-v^{\prime}(x) w(x)\right)=0$, then $C^{\prime}(y)=0$ and $F_{y}(x, y)=0$, which is excluded by assumption. Hence

$$
\phi_{x}(x, y) v(x)-\phi(x, y) v^{\prime}(x)=\phi_{x y}(x, y) \phi(x, y)-\phi_{y}(x, y) \phi_{x}(x, y) \text {. }
$$

Step 2: Differentiating equation (26) with respect to $y$ gives

$$
\phi_{x y}(x, y) v(x)-\phi_{y}(x, y) v^{\prime}(x)=\phi_{x y y}(x, y) \phi(x, y)-\phi_{y y}(x, y) \phi_{x}(x, y) \text {. }
$$

If

$$
\phi_{y}(x, y) \phi_{x}(x, y)=\phi_{x y}(x, y) \phi(x, y),
$$

then $\phi(x, y)$ is of the form $D(x) \cdot E(y)$ and again $\log \phi(x, y)$ is additively separable in $x$ and $y$ which contradicts the assumption. Hence the system (26) and (27) allows to recover $v$ :

$$
v(x)=\frac{\phi_{x y y} \phi^{2}+\phi_{y}^{2} \phi_{x}-\phi \phi_{x y} \phi_{y}-\phi \phi_{y y} \phi_{x}}{\phi_{x y} \phi-\phi_{y} \phi_{x}} .
$$

This defines $A(x)$ up to some multiplicative constant $a$, and generate testable conditions since the right hand side cannot depend on $x$. 
Step 3: In our case, $A(x)=B^{\prime}(x)$, so $B(x)$ is identified from $A(x)$ up to some additive constant $b$ :

$$
B(x)=a \bar{B}(x)+b
$$

where $\bar{B}(x)$ is known; $B(x)$ is thus identified up to an affine transform. Finally, since $w(x)=v(x) B(x)-B^{\prime}(x)=a\left(\bar{B}(x) v(x)-\bar{B}^{\prime}(x)\right)+b v(x)$, equation (24) becomes

$$
C(y)=a \frac{\phi_{x}\left(\bar{B} v-\bar{B}^{\prime}\right)-\phi\left(\bar{B}^{\prime} v+\bar{B} v^{\prime}-\bar{B}^{\prime \prime}\right)}{\phi_{x} v-\phi v^{\prime}}+b
$$

and $C(y)$ is identified up to the same affine transform.

CASE 2 (PARTICULAR CASE): $\log \phi(x, y)$ is additively separable in $x$ and $y$. Then $\frac{\partial^{2} \log \phi(x, y)}{\partial x \partial y}=0$; since

$$
\phi(x, y)=\frac{A^{\prime}(x) B(x)-A(x) B^{\prime}(x)}{A(x)} \frac{1}{C^{\prime}(y)}-\frac{A^{\prime}(x)}{A(x)} \frac{C(y)}{C^{\prime}(y)}
$$

this implies either

$$
\begin{aligned}
\frac{C(y)}{C^{\prime}(y)} & =\frac{N}{C^{\prime}(y)} \\
& \text { or } \\
A^{\prime}(x) B(x)-A(x) B^{\prime}(x) & =0 \\
A^{\prime}(x) & =0 \\
\frac{A^{\prime}(x) B(x)-A(x) B^{\prime}(x)}{A(x)} & =N \frac{A^{\prime}(x)}{A(x)}
\end{aligned}
$$

for some constant $N$. The first relation implies that $C(y)=N$, hence $F_{y}(x, y)=0$, a contradiction. The second, with the fact that $A(x)=B^{\prime}(x)$, implies that $B$ is exponential or linear. The third implies that $A(x)$ is constant and $B(x)$ linear. Finally, the fourth case gives

$$
A^{\prime}(x)(B(x)-N)=A(x) B^{\prime}(x)
$$


hence

$$
A(x)=a(B(x)-N)
$$

for some constant $a$. Since $A(x)=B^{\prime}(x)$, finally

$$
B(x)=\mu e^{a x}+N
$$

and $B(x)$ is exponential. Note that, in that case, identification does not hold. Indeed, if $F(x)=e^{x} /\left(e^{x}+C(y)\right)$ and $G(u)=a /\left(1+\left(\frac{1-u}{u}\right)^{a}\right)$, then

$$
G\left(\frac{e^{x}}{e^{x}+C(y)}\right)=\frac{a e^{a x}}{e^{a x}+C(y)^{a}}
$$

and the right-hand-side is also of the required form with $B(x)$ exponential with a different coefficient.

\section{References}

[1] Binmore, Ken, Ariel Rubinstein and Asher Wolinsky, 1986, "The Nash Bargaining Solution in Economic Modelling", The RAND Journal of Economics, Vol. 17, No. 2, pp. 176-188

[2] Bossert, Walter and Yves Sprumont, 2002, "Core Rationalizability in Two-Agent Exchange Economics", Economic Theory, vol. 200, pp. 777791.

[3] Bossert, Walter and Yves Sprumont, 2003, "Efficient and NonDeteriorating Choice", Mathematical Social Sciences, vol. 45, pp. 131142 .

[4] Carvajal, Andrés, 2002, "On Individually-Rational Choice and Equilibrium", PhD Thesis, Brown University.

[5] Carvajal, Andrés, Indrajit Ray and Susan Snyder, 2004, "Equilibrium Behavior in Market and Games: Testable Restrictions and Identification", Journal of Mathematical Economics, vol. 40, pp. 1-40.

[6] Chiappori, Pierre-André, 1988a, "Nash Bargained Household Decisions", International Economic Review, vol. 32, 791-796. 
[7] Chiappori, Pierre-André, 1988b, "Rational Household Labor Supply", Econometrica, vol. 56, pp.63-89.

[8] Chiappori, Pierre-André, 1991, "Nash Bargained Household Decisions : a Rejoinder", International Economic Review, 32 3, 1991, 761-62.

[9] Chiappori, Pierre-André, 1992, "Collective Labor Supply and Welfare", Journal of Political Economy, vol. 100, pp. 437-467.

[10] Chiappori, Pierre-André and Olivier Donni, 2004, "Nash-bargained Household Behavior", Manuscript.

[11] Chiappori, Pierre-André and Ivar Ekeland, 2003, "The Micro Economics of Group Behavior : Identification", Manuscript, University of Chicago.

[12] Chiappori, Pierre-André and Ivar Ekeland, 2004, "The Micro Economics of Group Behavior : General Characterization", Manuscript, University of Chicago.

[13] Coles, Melvyn G. and Andrew K.G. Hildreth, 2000, "Wage Bargaining, Inventories and Union Legislation", Review of Economic Studies, vol. 67, pp. 273-293.

[14] De Menil, Georges, 1971, Bargaining: Monopoly Power versus Union Power, MIT Press, Cambridge Mass.

[15] Farber, Henry S., 1986, "The Analysis of Union Behavior". In: O.C. Ashenfelter and R. Layard, Handbook of Labor Economics, vol. 2, pp. 1039-1089.

[16] Hamermesh, Daniel S., 1973, "Who 'Wins' in Wage Bargaining", Industrial and Labor Relations Review, vol. 26, pp. 1146-1149.

[17] Manser, Marilyn and Murray Brown, 1980, "Marriage and Household Decision-making", International Economic Review, vol. 22, pp. 31-44.

[18] McElroy, Marjorie and Mary-Jane Horney, 1981, "Nash-Bargained Household Decisions: Toward a Generalization of the Theory of Demand", International Economic Review, vol. 22, pp. 333-349. 
[19] McElroy, Marjorie and Mary-Jane Horney, 1990, "Nash-Bargained Household Decisions: Reply, International Economic Review, Vol. 31, No. 1, pp. 237-242

[20] Lundberg, Shelly and Robert A. Pollak (1993), "Separate Spheres Bargaining and the Marriage Market", Journal of Political Economy, vol. 101, pp. 988-1010.

[21] Myerson, Roger B., 1990, Game Theory, Harvard University Press.

[22] Nash, John, 1950, 'The Bargaining Problem', Econometrica, 28, 155-62.

[23] Ray, Indrajit and Lin Zhou, 2001, "Game Theory via Revealed Preferences", Games and Economic Behavior, vol. 37, pp. 415-424.

[24] Ray, Indrajit and Susan Snyder, 2003, "Observable Implications of Nash and Subgame-Perfect Behavior in Extensive Games", Working Paper 03-02, Department of Economics, Brown University.

[25] Roth, Alvin E. and Michael W.K. Malouf (1979), "Game-theoretic models and the role of information in bargaining ", Psychological Review, vol. 86, pp. 574-594.

[26] Rubinstein, Ariel, Zvi Safra and William Thomson (1992), "One the Interpretation of the Nash Bargaining Solution and its Extension to Non-expected Utility Preferences", Econometrica 60, 1171-1186.

[27] Siegel, Sidney and Lawrence E. Fouraker (1960), Bargaining and GroupDecision Making: Experiments in Bilateral Monopoly, MacMillan: NewYork.

[28] Sprumont, Yves, 2000, "On the Testable Implications of Collective Choice Theories", Journal of Economic Theory, vol. 101, pp. 437-456.

[29] Svejnar, Jan, 1980, "On Empirical Testing of the Nash-Zeuthen Bargaining Solution", Industrial and Labor Relations Review, vol. 33, pp. $536-542$.

[30] Svejnar, Jan, 1986, "Bargaining Power, Fear of Disagreement, and Wage Settlements: Theory and Evidence from U.S. Industry", Econometrica, vol. 54, pp. 1055-1078. 
[31] Thomson, William, 1994, "Cooperative Models of Bargaining". In: R.J. Aumann and S. Hart (eds), Handbook of Game Theory, vol. 2, pp. 12371277.

[32] Xu, Yongsheng and Lin Zhou, 2004, "Rationalization of Collective Choice Functions by Games with Perfect Information", Manuscript, Arizona Sate University.

[33] Zhou, Lin, 1997, "Revealed Preferences and Status Quo Effect", Manuscript, Duke University.

[34] Zhou, Lin, 2002, "Testable Implications of Nash Equilibrium Theory", Manuscript, Arizona State University. 